\title{
Determination of the Raman Polarisabilities of Optical Phonons in Lithium Niobate Uniaxial Single Crystal.
}

\author{
N. Djiedeu ${ }^{1}$, B. Mohamadou ${ }^{1}$, P. Bourson ${ }^{2}$ and M. Aillerie $^{2}$ \\ ${ }^{I}$ CEPAMOQ, University of Douala P.O.Box 8580,Cameroon,djienico@yahoo.fr; ballo_mohamadou@yahoo.fr \\ ${ }^{2}$ LMOPS, UMR CNRS 7132, Université Paul Verlaine - Metz et Supélec, 2, rue E. Belin, 57070 Metz, France
}

\begin{abstract}
Raman scattering is an excellent tool to probe matter for the research of its dynamical properties particularly in the aim of its integration in non linear optical applications. However, only few efforts have been devoted to the connection of the Raman scattering measurements with the nonlinear optical properties of inorganic materials such as $\mathrm{LiNbO}_{3}(\mathrm{LN})$ and $\mathrm{LiTaO}_{3}$ (LT). Such a relation requires at the first glance the determination of the Raman polarisability tensor elements whose absolute or relative measurement is experimentally very difficult. In the present study, we described a simple method based on crystal rotation, which leads us to measurements of the scattering efficiencies and their relative phase. More specifically, we characterized a congruent lithium niobate and we recorded its Raman spectra in the backscattering geometries e.g. for the following configurations $(\mathrm{x}(\mathrm{yy}) \underline{\mathrm{x}}, \mathrm{x}(\mathrm{yz}) \underline{\mathrm{x}}, \mathrm{x}(\mathrm{zy}) \underline{\mathrm{x}}, \mathrm{x}(\mathrm{zz}) \underline{\mathrm{x}})$. The measured spectra were fitted by using the Lorentz or Fano functions. The parameters deduced from the fit are presented as functions of rotation angles of crystal. This allows us to validate the selection rules and to determinate the relative phase and as the absolute values of the Raman tensor elements.
\end{abstract}

\section{Introduction}

Raman spectroscopy is an important tool for characterization of intrinsic properties of numerous materials. Particularly, the Raman scattering efficiencies and their relative phase are important features in the study of the dynamics of superconductors ${ }^{1}$ such as $\mathrm{SmBa}_{2} \mathrm{Cu}_{3} \mathrm{O}_{7-\delta}$. Alternatively, the values of Raman polarisabilities reveal as crucial parameters involved in the determination of the electro-optic $(\mathrm{E} / \mathrm{O})$ coefficients from Raman spectroscopy data ${ }^{2}$. Unfortunately, the experimental determination of such quantities is difficult because it needs a lot of cautious including mainly optical characterization of sample and components used in the experiment. Several methods ${ }^{3}$ are used for the experimental determination of absolute or relative Raman scattering tensor elements. However owing to the lot of constraints required by the absolute measurements, comparative method is often preferred even though this latter must be accompanied with internal or external standard. Many works devoted to the measurement of the Raman efficiency were absolutely or relatively performed on solvent ${ }^{4}$. Besides, the zinc-blend structure such as cuprous chloride ${ }^{5}$ or gallium phosphide ${ }^{6}$ which exhibit a few Raman lines (no more than five peaks) has been studied experimentally as well as theoretically. More specifically, absolute measurements on GaN have been carried out ${ }^{7}$, using the method of translation of sample or alternatively the movement of the microscope objective. The advantage of the studies focussed on diatomic substances dwell on the simple character of their structure. Meanwhile, only few attempts were devoted to solid substances with more than two atoms such as lithium niobate ${ }^{8}\left(\mathrm{LiNbO}_{3}\right.$ or $\mathrm{LN})$ and lithium tantalate ${ }^{8}\left(\mathrm{LiTaO}_{3}\right.$ or LT) on the one hand and lithium meta gallate $\left(\mathrm{LiGaO}_{2}\right)$ on the other hand. Both studies used the $992 \mathrm{~cm}^{-1}$ Raman line of benzene used as the reference. Since the first principle of band structure calculations leading to the prediction of Raman efficiencies for dielectric compound with more complex structures are extremely difficult to carry out, only the experimental counterpart remains the way to be adopted.

The purpose of the present work is (1) the description of a new and simple comparative method based on rotating crystal leading consequently to the determination of Raman 
efficiencies and their relative phases and (2) the derivation of the general form of the Raman tensor yielding to the selection rule angle dependent e.g. for any angular position of polarisers, the integrated intensities of the Raman spectrum can be clearly analysed. The determination of the relative phases as well as the theoretical derivation of Raman polarisabilities tensor are news features which reveals the advantage of this method over others comparative methods ${ }^{(8,9)}$. Rotating crystal has also the advantage of moving only the sample support instead of rotating simultaneously the polarisers. Additionally, this method can also be extended to others point groups such as $\mathrm{C}_{2 \mathrm{v}}, \mathrm{C}_{4 \mathrm{v}}, \mathrm{C}_{6 \mathrm{v}}$. To check the reliability of the approach as presented in this paper, the derived equations of the fundamental method (section II) will be applied to the characterization of a congruent LN crystal and compare the so-obtained Raman efficiencies results to those already published ${ }^{8}$.

\section{Theoretical foundations of the method}

Owing to the proportionality relation ${ }^{7}$ between the Raman cross section and the area under the studied peak for initial parallel polarisation filter (yy), it has been established:

$\sum_{y y}^{A(\theta)} \propto G\left(\omega_{m}\right)\left(|a|^{2} \cos ^{4}(\theta)+|b|^{2} \sin ^{4}(\theta)+(1 / 2)|a||b| \cos \varphi_{b a} \sin ^{2}(2 \theta)\right)$

Similarly to $\mathrm{A}_{1}$ mode and for initial parallel polarizations, Eq.1 yields:

$\sum_{y y}^{E_{2}(\theta)} \propto G\left(\omega_{m}\right)\left(|c|^{2} \cos ^{4}(\theta)+|d|^{2} \sin ^{2}(2 \theta)+2|c||d| \cos ^{2}(\theta) \sin (2 \theta) \cos \left(\varphi_{d c}\right)\right)$

a,b,c,d are the Raman tensor elements in the $\mathrm{C}_{3 \mathrm{v}}$ point group ${ }^{10}, \varphi_{b a}, \varphi_{d c}$ are the relative phases of the Raman tensor elements corresponding to the $A_{1}$ and $E_{2}$ symmetric types and is the area under the Raman line $\mathrm{m}$. In Eqs 1 and $2 \mathrm{G}\left(\omega_{\mathrm{m}}\right)$ is defined as

$G\left(\omega_{m}\right)=\frac{\hbar\left(\omega_{i}-\omega_{m}\right)^{4}\left(n_{m}+1\right)}{32 \pi^{2} c^{4} \omega_{m}}$

where $\omega_{i}$ and $\omega_{m}$ are the laser and phonon energies, $\mathrm{n}_{\mathrm{m}}+1$ is the Stokes phonon occupation

Eqs. 1 and 2 will be used in the fit procedure to determine the fundamental areas proportional to the Raman tensor elements a, b, c, d. More precisely, at a given rotation angle of the crystal, a Raman peak is considered as being the result of the interference of the fundamental.

\section{Experimental results and discussion}

A congruent lithium niobate crystal obtain by Czochralski method was oriented following the (100), (010), (001) dielectric axes. The Raman spectrum has been recorded at room temperature. A Labram Jobin-Yvon spectrometer was used for micro-Raman and confocal luminescence experiments. Radiation of an argon ion laser $(514.5 \mathrm{~nm})$ is focused trough a microscope objective (50x/NA 0.7) on the crystal surface. A back-scattering scheme is used to detect the Raman signal trough a confocal pinhole, allowing to extract the light scattered from an extremely small volume (diameter is $1.6 \mu \mathrm{m}$ and depth is $4 \mu \mathrm{m}$ with the microscope objective x100) of the crystal. So, the detection of Raman or luminescence signals from a very small focal area can be performed, where extremely high intensities are available even at small input powers. The sample is tied on a rotation stage allowing the crystal to move degree per degree. The measurement of both (TO) $A_{1}$ and $E_{2}$ symmetry species was done with initial polarisation filter $(y y)$ when the propagation of the incident and scattered waves are along the $\mathrm{x}$ axis e.g. $\mathrm{x}(\mathrm{yy}) \underline{\mathrm{x}}$. The spectra were recorded with angular step of $10^{\circ}$ without internal or external reference.

Fig. 1 presents the room temperature Raman spectra recorded at $0^{\circ}, 40^{\circ}$ and $90^{\circ}$ angular positions of the crystal, the spectrum at $40^{\circ}$ is characteristic of those obtained from 30 to $60^{\circ}$. 


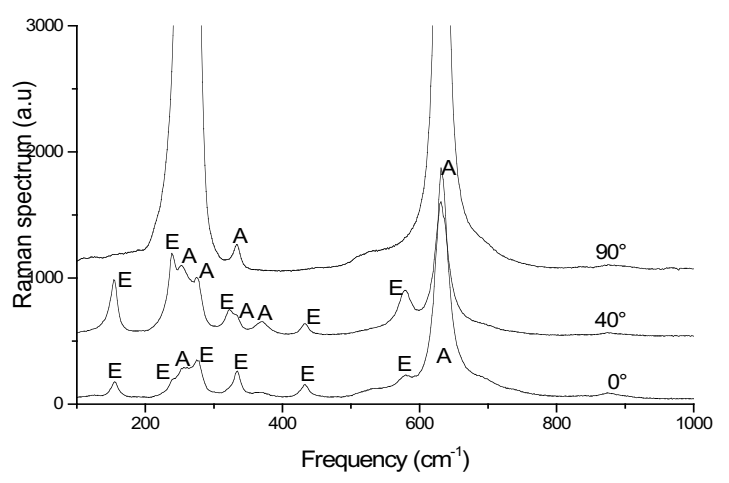

Fig 1 : Raman Spectra of pure $\mathrm{LiNbO}_{3}$ recorded as function of polarisation rotation angles for parallel polarisations

Despite of the recording temperature $(300 K)$, some $E$ modes are clearly observed without the notified overlapping ${ }^{6}$. From 30 to $60^{\circ}$, one can observe several lines which are clearly attributed to the modes of $E$ symmetry specie. On the contrary the peaks corresponding to the $\mathrm{A}_{1}$ modes disappear in this angular range. Such behaviours are merely the consequence of the selection rules described by (1) and (2).

For the limiting cases e.g. case of $0^{\circ}$ and $90^{\circ}$ corresponding to $x(y y) \underline{x}$ and $x(z z) \underline{x}$ geometrical configurations, one can retrieve the classical selection rule giving at $0^{\circ}$ the spectra of the $a(T O)$ and $c(T O)$ in the $x(y y) \underline{x}$ on the one hand and at $90^{\circ}$ the $b(T O)$ of the $\mathrm{A}_{1}$ modes on the other hand. All the recorded spectra were fitted either with Lorentz function for the symmetric Raman profile or with Fano ${ }^{12}$ function for the asymmetric one. This latter case is only applied to the $633 \mathrm{~cm}^{-1}$ Raman line for the present study. The parameters deduced from this first series of fit are the resonant TO frequency, the damping and the high of peak and are in turn used for the calculation of the area of the studied peak according to the approximation of isosceles triangles. As stated above, the measurement was done without internal or external reference. Since one of the aims of the present work is the validation of the rotating crystal method, we have chosen as reference in our measured spectra the structure at $369 \mathrm{~cm}^{-1}$ which appears for the whole range of the measurement angles. As a consequence, Fresnel, refraction, absorption and others corrections were assumed to implicitly compensate and can be therefore neglected. In fact a reference sample must be chosen to fulfil the conditions required by comparative method.

All the spectra were then normalised by dividing their computed areas by the area under the c(TO) mode measured at $369 \mathrm{~cm}^{-1}$ here considered as our reference. The second step of the fit is aimed to the calculations of the areas associated with the Raman tensor elements $a, b, c$ and $d$. By using mathematical handlings, we have reduced the 3 parameters $\left(a, b, \varphi_{b a}\right)$ for $\mathrm{A}_{1}$ modes and $\left(c, d, \varphi_{d c}\right)$ for $E$ modes to 2 parameters by solving exact values of the phase differences $\left(\varphi_{b a}, \varphi_{d c}\right)$. Finally, the polarisabilities of the studied lines are deduced from the value of the reference efficiency ${ }^{8}\left(0.014 \times 10^{-7} \mathrm{~cm}^{-1} \mathrm{sr}^{-1}\right.$ at $\left.374 \mathrm{~cm}^{-1}\right)$. The second step of fit is shown in figure 2 through two examples. Figure 2 a described the angular behaviour of the areas under the $633 \mathrm{~cm}^{-1}$ peak according to (1) while in figure $2 \mathrm{~b}$ is presented the enhancement of the $369,5 \mathrm{~cm}^{-1}$ line of the $E_{2}$ symmetry type using (2). We have reported in table I, the calculated Raman polarisabilities for the above mentioned examples (figure 2) in comparison with the published results concerning the $\mathrm{LiNbO}_{3}$ crystal 

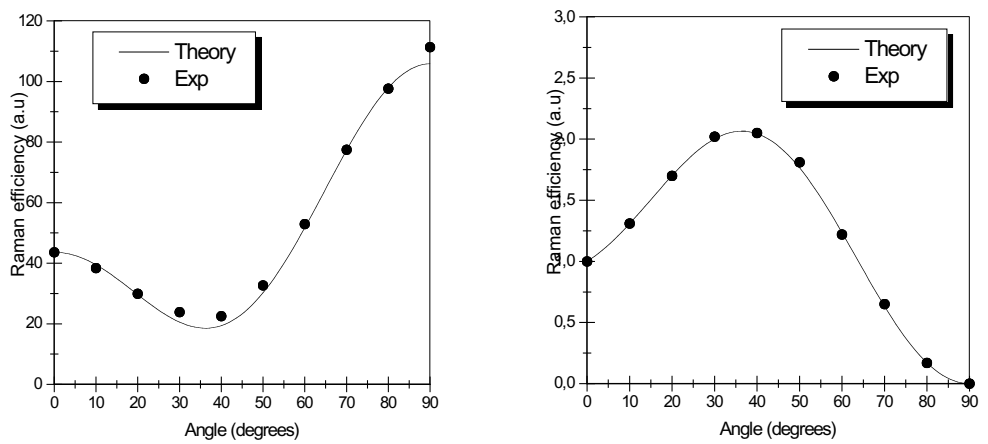

Fig $2:$ (a) Areas of the 633 $\mathrm{cm}^{-1}$ of the TO phonon of the $\mathrm{A}_{1}$ symmetry type following (1).

(b) Areas of the $369 \mathrm{~cm}^{-1}$ line associated to the TO phonon of the $E_{2}$ mode fitted according (2)

\begin{tabular}{|c|c|c|l|l|}
\hline $\begin{array}{c}\text {. Component } \\
\text { vibration }\end{array}$ & $\begin{array}{c}\text { Frequency } \\
\left(\mathrm{cm}^{-1}\right)\end{array}$ & $\begin{array}{c}\text { Phase } \\
\text { difference }\end{array}$ & \multicolumn{2}{|c|}{$\begin{array}{c}\text { Raman } \\
\text { efficiency }\left(10^{-7} \mathrm{~cm}^{-1} \mathrm{sr}^{-1}\right)\end{array}$} \\
\hline $\mathrm{E}(\mathrm{TO})^{\mathrm{a}}$ & 369,5 & $\varphi_{d c}=75^{\circ}$ & $|c|=|c|$ & $|d|=1,17$ \\
\hline $\mathrm{E}(\mathrm{TO})^{\mathrm{b}}$ & 374.0 & - & $|c|=0,014$ & $|d|=1,33$ \\
\hline $\mathrm{A}_{1}(\mathrm{TO})^{\mathrm{a}}$ & 633.3 & $\varphi_{b a}=114,6^{\circ}$ & $|a|=8,30$ & $|b|=12,93$ \\
\hline $\mathrm{A}_{1}(\mathrm{TO})^{\mathrm{b}}$ & 639.0 & - & $|a|=6,57$ & $|b|=14,42$ \\
\hline
\end{tabular}

Table 1 : Comparison of the present results ${ }^{a}$,, with those of Ref. 8.

It can be noticed that the early experiments ${ }^{8}$ were performed under the radiation of the $488 \mathrm{~nm}$ line of ion argon laser. As can be observed, our preliminary results are in good agreement with ref. (8).

\section{Conclusion}

The present work has pointed out a general method to experimentally determine the module and the phase difference between the Raman tensor elements of the polar modes. In addition to the generalisation of selection rule, the method has been successfully applied to the LN Raman spectra in the $x(..) \underline{x}$ geometrical configurations and is applicable to others point groups. The precision of the fit may be improved by increasing the number of measurements.

\section{References}

1. T. Strach,J. Brunen,B. Lederle,J. Zegenhagen, M. Cardona, "Determination of the phase difference between the Raman tensor elements of the $A_{\text {lg }}$ like phonons in $\mathrm{SmBa}_{2} \mathrm{Cu}_{3} \mathrm{O}_{7-\delta}$ ", Phys. Rev B 571292 (1998)

2. R. Loudon, "The Raman effect in crystals", ADV. Phys. 13, 52, 423-482 (1964)

3. N. Biswas and S. Umpathy "Simple Approach to Determine Absolute Raman Cross Section Using an Optical Parametric Oscillator”, Applied Spectroscopy, 52, 4, 496 - 499 (1998)

4. K. T. Schomacker, J. K. Delaney, and P. M Champion, "Measurements of the absolute Raman cross sections of benzene", J. Chem.Phys. 85, 8, 4240 (1986)

5. I. P. Kaminow and E. H. Turner, "Temperature Dependence of Raman Scattering and the Electro-optic Properties of CuCl”, Phys.Rev.B 5, 1564 (1972)

6. V.S. Gorelik, , O.G. Zolotukhin, and M.M. Sushchinskiy, "Effective Raman Scattering Cross-Section and Its Coupling With Nonlinear Coefficients in Single Crystals of Gap", ZhPS 28, 3, 495-498 (1978)

7. I.Loa, S. Gronemeyer, C. Thomsen, O. Ambacher, D. Shikora and D. J. As, "Comparative Determination of Absolute Raman Scattering Efficiencies and Application to GaN”, J. Raman Spectr. 29, 291-295 (1998)

8. I.P. Kaminow and W.D Johnston, Jr., "Quantitative Determination of Sources of the Electro-Optic Effect in $\mathrm{LiNbO}_{3}$ and $\mathrm{LiTaO}_{3}$ ”, Phys. Rev. 160, 519 (1967) and 178, 1528 (Errata) (1969)

9. P. Knoll and H. Kuzmany, "Nonlinear Optical Properties and Signs of the Raman Tensor for LiGaO ${ }_{2}$ " Phys. Rev. B29, 4, 2221 (1984)

10. A.F. Penna, A. Chaves, P. da R. Andrade, and S. P. S. Porto, "Light scattering by lithium tantalate at room temperature", Phys.Rev.B, 13, 4907 (1976)

11. W.D Johnston, Jr, "Nonlinear Optical coefficients and the Raman scattering Efficiencies from LO and TO Phonons in Acentric crystals", Phys. Rev. B1, 3494 (1970)

12. U. Fano, Phys. Rev. 124, 1866 (1961) 